\begin{tabular}{|c|ccc|c|}
\hline $\begin{array}{c}\text { PORT SAID ENGINEERING RESEARCH JOURNAL } \\
\text { Faculty of Engineering - Port Said University } \\
\text { No.1 }\end{array}$ & March 2021 & pp:49-58
\end{tabular}

\title{
Fully Generalized Spatial Modulation utilizing Transmit Antenna Grouping
}

\author{
Islam E. Shaalan ${ }^{1}$, Saied Dawod ${ }^{2}$, and Sherif M. Abuelenin ${ }^{3}$
}

Received: 16 November 2020; Accepted: 28 January 2021

\begin{abstract}
One of the recently introduced multiple-input multiple-output (MIMO) systems is the spatial modulation (SM). SM wireless communication system achieves an enhanced power efficiency, low error rate, low complexity at receiver, and complete inter-channel interference (ICI) elimination. On the other side, SM suffers from the dramatic increase in the number of required transmit antennas to achieve a small improvement in data rate and low spectral efficiency when compared with multiplexing MIMO (Mux-MIMO). The logarithmic increasing in data rate of SM scheme became linear thanks to the fully generalized spatial modulation scheme (F-Gen-SM). However, the F-Gen-SM achievable data rate is still low when compared with Mux-MIMO. Grouping generalized spatial modulation scheme (Gr-Gen-SM) tries to compromise between the advantage of SM and the high spectral efficiency of Mux-MIMO. Gr-Gen-SM achieves high spectral efficiency but loses most of the SM advantages. This paper introduces a new F-Gen-SM scheme utilizing the idea of dividing transmit antennas into groups (F-Gr-Gen-SM). The proposed F-Gr-Gen-SM scheme acquire its spectral efficiency by two consecutive steps. At first, the transmitting antennas are divided into a number of groups. Each group is considered as a separate F-Gen-SM system. Then the different data bits from all groups are multiplexed to be transmitted to receiver side at the same time as a Mux-MIMO system. Thus, proposed scheme provides an improvement on data rate while preserving the advantages of SM as much as possible. Simulation results show that the proposed scheme can improve the performance of the system by about $6.5 \mathrm{~dB}$ as compared with F-Gen-SM. Also, the proposed scheme is the best one in rationalizing the number of transmit antennas as compared with both F-Gen-SM and Gr-Gen-SM schemes.
\end{abstract}

Keywords: SM; MIMO; data rate; ICI; Mux-MIMO; F-Gen-SM; Gr-Gen-SM; F-Gr-Gen-SM.

\section{INTRODUCTION}

One of the encouraging technologies for wireless communication systems, spatial modulation (SM) [1] was recently proposed for enhancing the data rate of the system. The SM system consists of multiple antennas at both the transmitting and receiving sides. Thus, SM is considered a multiple-input multiple-output (MIMO) system [2]. Although SM has multiple transmit antennas, a single antenna is activated at the transmitting side at any time instant. Thus, virtually, SM is a single-input multiple-input (SIMO) system in its operation idea. To clearly understand the SM scheme, the idea of space shift keying (SSK) [3], [4] must be explained first. SSK scheme implicitly transmits the data when choosing an index of transmit antenna out of all transmit antennas to be activated without modulating the desired symbol to be transmitted. This symbol, which is called the spatial

\footnotetext{
1 Electrical Engineering Department, Faculty of Engineering, Port Said University, Port Fouad, Port Said, Egypt, email: is_shaalan@eng.psu.edu.eg

2 Communication and Electronic Engineering Department, High Institute of Engineering and Technology in El-Arish, El-Arish, Egypt, email: engsaied@hotmail.com

3 Electrical Engineering Department, Faculty of Engineering, Port Said University, Port Fouad, Port Said, Egypt, email: s.abuelenin@eng.psu.edu.eg
}

DOI: $\underline{10.21608 / \text { pserj.2021.47715.1070 }}$ symbol, is responsible for determining which transmit antenna will be activated. SM exploits both the conventional digitally modulated symbol and the spatial symbol to be transmitted at the same time.

Hence, the data rate of the SM is higher than both the SSK and single-input single-output (SISO) systems. Therefore, the SM spectral efficiency is calculated from the following formula [1]:

$$
\eta_{S M}=\log _{2}(M)+\log _{2}\left(N_{t}\right)
$$

where $M$ indicates the constellation size of the quadrature amplitude modulation (QAM) and $N_{t}$ is the number of antennas at the transmit side.

When SM is compared with multiplexing multipleinput multiple-output (Mux-MIMO), the data rate is extremely low especially for large number of transmitting antennas. On the other hand, SM eliminates the inter-channel interference (ICI) as at any time only one transmit antenna is activated, hence, there is only one channel path from the active antenna on the transmitting side to each antenna on the receiving side. While each transmits antenna of MIMO system needs an RF chain and the overall RF chain increases the system cost overwhelming, SM needs one RF chain with a splitter to steer this RF chain to the active transmit antenna for each time, this reduces the cost of the SM system. Also, the transmitted power for SM is concentrated on the active transmit antenna at any time instead of weakening it by dividing it into all the transmit antennas as in Mux-MIMO. Finally, the complexity of the SM system decreased when compared with Mux-MIMO. 
On the other hand, the disadvantages of SM are represented in two points. The first disadvantage of SM is the number of transmit antennas which is restricted to a base-two number. The second disadvantage of SM is the increased number of transmit antennas required to achieve a limited spectral efficiency.

As for generalized space shift keying (GSSK) [5], [6], generalized spatial modulation (Gen-SM) technique was proposed [7]-[11]. In Gen-SM, multiple antennas are activated at the transmitter side. Increasing the number of activating transmit antennas means more possible active antennas combinations.

For example, in an SM system with 8 transmit antennas, any single antenna may be activated, giving eight different possibilities. While, for Gen-SM, assuming $N_{t}=8$ while activating 4 transmit antennas, the possible active antennas subset combinations can be calculated as $\left(\begin{array}{l}N_{t} \\ N_{u}\end{array}\right)=\left(\begin{array}{l}8 \\ 4\end{array}\right)=70$. Where $N_{u}$ is the number of active transmit antennas.

Therefore, the spatial bits of Gen-SM is $\left|\log _{2}\left(\begin{array}{l}N_{t} \\ n_{u}\end{array}\right)\right|=$ $\left\lfloor\log _{2}\left(\begin{array}{l}8 \\ 4\end{array}\right)\right\rfloor=6$ bits, while for SM is $\log _{2}\left(N_{t}\right)=\log _{2}(8)=$ 3. In other words, the spatial bits of the Gen-SM are two times as compared with the SM.

Note that, compared with the SM scheme, Gen-SM not only improves the data rate, but also $N_{t}$ can equal to any integer number.

Hence, the data rate of the Gen-SM can be calculated as [7]-[9]

$$
\eta_{\text {Gen-SM }}=\log _{2}(M)+\left\lfloor\log _{2}\left(\begin{array}{c}
N_{t} \\
N_{u}
\end{array}\right)\right\rfloor
$$

The Fully generalized spatial modulation scheme (FGen-SM) was proposed [12], [13], [14] to overcome the problem of logarithmic increase in the SM spectral efficiency. F-Gen-SM was proposed by changing $N_{u}$, which leads to increase in the number of possible active antennas combination. Hence, the F-Gen-SM data rate is calculated as follows [12], [13], [14]:

$$
\begin{aligned}
\eta_{F-\text { GenSM }} & =\log _{2}(M)+\left\lfloor\log _{2}\left(\sum_{j=1}^{N_{t}}\left(\begin{array}{c}
N_{t} \\
j
\end{array}\right)\right)\right\rfloor \\
& =\log _{2}(M)+\left(N_{t}-1\right)
\end{aligned}
$$

Although the F-Gen-SM spectral efficiency is improved as compared with SM, it is still very limited compared with Mux-MIMO.

Grouping generalized spatial modulation scheme (GrGen-SM) was proposed in [15] to achieve high spectral efficiency. All transmitting antennas of the Gr-Gen-SM scheme are equally divided into a number of antenna groups. Each group is considered as a Gen-SM scheme while the spectral efficiencies of all groups are multiplexed as a Mux-MIMO system. Hence, the spectral efficiency of the system is highly increased, while losing almost all advantages of the SM. The number of required RF chains will increase, as one RF chain is needed for each group of antennas. Hence, the Gr-Gen-SM data rate is calculated as follows [15]:

$$
\eta_{G r-G e n-S M}=N_{G}\left(\log _{2}(M)+\log _{2}\left(\begin{array}{c}
N_{G t} \\
N_{u}
\end{array}\right)\right)
$$

where $N_{G}$ stands for the number of groups and $N_{G t}$ is the number of transmit antenna in each group.

Gr-Gen-SM scheme [15] sided with the mux-MIMO more than SM scheme where only two transmit antennas are considered for each group. Maximizing the number of groups means an equally large number of RF chains are needed instead of a single RF chain as in the previous space modulation techniques (SMTs). This large number of RF chains means high cost of the system. Also, this causes more inter-channel interference due to the transmission of different data bits through the different groups. As for the transmitted power, the number of activated antennas at the transmitting side for Gr-Gen-SM equals to the number of antenna groups. Hence, to achieve maximum data rate, the transmitted power for each active transmit antenna is decayed.

To alleviate all these problems, we propose a new scheme, namely, the fully generalized spatial modulation utilizing transmit antenna grouping (F-Gr-Gen-SM), that compromises between the advantages of $\mathrm{SM}$ and the high data rate of Gr-Gen-SM.

The remainder of this paper is organized as follows. Section 2 presents F-Gr-Gen-SM system model. Section 3 presents the computational complexity analysis of the F-Gr-Gen-SM scheme. Section 4 presents the simulation results. Finally, the paper is concluded in Section 5.

\section{FULLY GENERALIZED SPATIAL MODULATION SCHEME UTILIZING TRANSMIT ANTENNA GROUPING}

Figure. 1 describes the system model of the proposed scheme. The transmit antennas are grouped into a number of groups $\left(N_{G}\right)$ each compromises a number of antennas $\left(N_{G t}\right)$ and applies the F-Gen-SM scheme.



FIGURE 1. The Fully -Generalized Spatial Modulation Utilizing Transmit Antenna Grouping System Model. 
At any time instant, the desired data bits to be conveyed is divided into a number of parts equal to the number of antenna groups $\left(N_{G}\right)$. Then, each part of bits is separated into two different bits sections. The digitally modulated data bits which equal $\log _{2}(M)$ bits, constitute the first section of bits. This part of the bits defines the modulated signal constellation symbol, which is modulated using M-order of quadrature amplitude modulation (M-QAM). The second part of the bits, which contains includes a number of bits equal to $\left(N_{G t}-\right.$ 1 ), is named the spatial bits. The spatial bits part is used to select a subset of antennas to be activated for transmitting the data constellation symbol. In the F-GenSM scheme, the active transmitting antennas may be a single, multiple, or all transmit antennas. The main difference between F-Gen-SM and Gen-SM scheme is that Gen-SM activates a fixed number of transmitting antennas. In F-Gen-SM [13], [14], varying the number of activated transmit antennas results in reduced searchspace for the maximum-likelihood detection, hence, reducing the probability of error.

Finally, the modulated symbols are multiplexed and transmitted over the chosen antennas as a MIMO system to achieve a high data rate. Thus, the achievable spectral efficiency of F-Gr-Gen-SM can be calculated as follows:

$$
\eta_{F-G r-G e n S M}=N_{G}\left(\log _{2}(M)+\left(N_{G t}-1\right)\right)
$$

The principle of the F-Gen-SM transmission is illustrated in Table 1, which is applied in each group of our proposed scheme. For example, a F-Gr-Gen-SM scheme with two antenna groups is supposed to achieve eight bits per channel use $(8 \mathrm{bpcu})$ spectral efficiency using $N_{t}=6$. Note that, this spectral efficiency can be achieved by the F-Gen-SM scheme using 7 transmit antennas. Suppose that a $\left[\begin{array}{llllllll}1 & 0 & 1 & 1 & 1 & 0 & 1 & 0\end{array}\right]$ data bits sequence is desired to be transmitted at a time. At first, these bits are partitioned into a number of bits part equal to $N_{G}$.

First bits section [ $\left.\begin{array}{llll}1 & 0 & 1 & 1\end{array}\right]$ is matched to the first transmit antenna group which divides this bits into two parts. The first part [ 10$]$ includes the digitally modulated data bits which extract the data constellation symbol $S^{1}$. The other part [ 11 ] is the spatial bits which are responsible for mapping the data symbol $S^{1}$ to the subset of transmitting antenna $\left(T_{X_{1}}\right.$ and $\left.T_{X_{2}}\right)$.

The second bits part [ $\left[\begin{array}{llll}1 & 0 & 1 & 0\end{array}\right]$ also matched to the second transmitting antenna group which divides these bits into two parts. The first bits part [ 10 ] includes the digitally modulated data bits which extract the data constellation symbol $S^{2}$. The other part of bits [ 10 ] contains the spatial bits which are responsible for mapping the data symbol $S^{2}$ to the transmit antenna $T_{X_{3}}$. Finally, both $S^{1}$ and $S^{2}$ data symbols are multiplexed to be transmitted over their chosen transmit antennas at the same time as a MIMO system.

For the $l$-th group, each $m$-th selected antenna transmits the signal $S_{m}^{(l)}, \quad l=\left\{1,2, \ldots, N_{G}\right\}, \quad m=$ $\left\{1,2, \ldots, N_{G t}\right\}$.

Hence, the proposed F-Gr-Gen-SM transmission vector in its final form $x \in C^{N_{t} \times 1}$ is $x=$ $\left[\begin{array}{lllll}S^{1} S^{1} & 0 & 0 & 0 & S^{2}\end{array}\right]^{T}$. Then this vector is conveyed over uncorrelated fading channel $\boldsymbol{H} \in C^{N_{r} \times N_{t}}$ and suffers from an additive white Gaussian noise (AWGN) $n \in$ $C^{N_{r} \times 1}$ having independent Gaussian distributed real and imaginary parts and is expressed as $\sim C N\left(0, \sigma_{n}^{2}\right)$, where $N_{r}$ stands for the number of receive antennas.

TABLE 1. F-Gen-SM example for achieving 4 bpcu using $M=4$ and $N_{t}=3$.

\begin{tabular}{|c|c|c|c|c|}
\hline \multicolumn{3}{|c|}{ Block of Bits } & \multicolumn{3}{c|}{ Transmitted Data } \\
\hline Data Bits & Spatial Bits & $T_{x 1}$ & $T_{x 2}$ & $T_{x 3}$ \\
\hline$b_{1} b_{2}$ & 00 & $S$ & - & - \\
\hline$b_{1} b_{2}$ & 01 & - & $S$ & - \\
\hline$b_{1} b_{2}$ & 10 & - & - & $S$ \\
\hline$b_{1} b_{2}$ & 11 & $S$ & $S$ & - \\
\hline Finally & $y=h_{l} S+n$
\end{tabular}

where $y \in C^{N_{r} \times 1}$ is the received vector of the proposed scheme on the receiving side.

And

$$
h_{l}=\sum_{c=1}^{N_{v}} h_{l c}
$$

where $h_{l}$ is the summation of the channel columns of the active transmitting antennas that utilized to data symbol transmission, $h_{l c}$ is the $c$-th columns of the channel, and $N_{v}=N_{u 1}+N_{u 2}+\cdots+N_{u g}+\cdots+N_{u G}$,

where, $N_{u g}=1,2, \ldots,\left\lceil\frac{N_{G t}}{2}\right\rceil$ is $N_{u}$ at a $g$-th transmit antenna group.

On the receiver side of the system, we assume that the channel state information (CSI) is perfectly known [6], [9].

Then, to obtain the optimum performance of the F-GrGen-SM, a maximum likely-hood (ML) decoder is assumed to be utilized. Thus, the activated antenna index and data symbol can be estimated as follows:

$$
\left[\tilde{d}_{a}, \tilde{S}\right]=\arg \min _{d_{a}, S}\left\|y-h_{l} S\right\|^{2}
$$

where $\tilde{d}_{a}$ and $\tilde{S}$ are the estimated transmitting antenna index and data symbol respectively, which are used to recover transmitted bits.

\section{COMPUTATIONAL COMPLEXITY ANALYSIS}

By counting the overall real operations number required at the ML decoders of any SMT system, the computational complexity $\left(\Gamma_{C}\right)$ can be calculated. Note that, one complex multiplication equals 4 real multiplications.

For a MIMO system, $4 N_{t} N_{r}$ operations are required for the multiplication between $h_{l c} S$ and the square operation requires $4 N_{r}$ operations which are repeated over $2^{\eta_{\text {MIMO }}}$ possible symbols. Thus, the number of operations that are required for a MIMO system is expressed as [19]:

$$
\Gamma_{C_{M I M O}}=4 N_{r}\left(N_{t}+1\right) 2^{\eta_{M I M O}}
$$

For the conventional SM case [1], to calculate $\left\|y_{i}-h_{l i} S\right\|^{2}$, two complex multiplications (8 real multiplications) are required in the ML decoder. To calculate the square norm, four real multiplications are needed and another four multiplications are needed to 
calculate the complex multiplication between $h_{l c} S$. These operations are repeated for each received antenna. Then it is repeated for each bit ( $2^{\eta_{S M}}$ bits). [19]

Therefore, the $\Gamma_{C}$ of the conventional SM is given by

$$
\Gamma_{C_{S M}}=8 N_{r}(2)^{\eta_{S M}}
$$

Similarly, the Gen-SM computational complexity can be calculated. As Gen-SM activating multiple transmit antennas, calculating $h_{l c}$ in the $M L$ decoder requires $\left(\mathrm{N}_{u}-1\right)$ complex summations [19], i.e., the number of real summations equals $2(N u-1)$. Therefore, the complexity of Gen-SM is calculated as follows:

$$
\Gamma_{C_{G e n-S M}}=8 N_{r}(2(N u-1))(2)^{\eta_{G e n-S M}}
$$

In the F-Gen-SM, determining $h_{l c}$ requires ( $\left\lceil\frac{N_{t}}{2}-\right.$ 1) complex summations at most for all $N_{t}$ equals at least 3 transmit antennas. Therefore, the complexity of the FGen-SM scheme is calculated as follows [12], [13]:

$$
\Gamma_{C_{F-G e n-S M}}=8 N_{r}\left(\left(2\left\lceil\frac{N_{t}}{2}-1\right\rceil\right)\right)(2)^{\eta_{F-G e n-S M}}
$$

Regarding the Gr-Gen-SM, the number of required complex summations for each group is $(N u-1)$ (i.e. $2(N u-1)$ real summations). For all groups, the total number of operations of the system which is considered as a MIMO system is $\left(4 N_{G} N_{r}(2(N u-1))+4 N_{r}\right)$. This number of operations is required for a single bit transmission. Thus, the overall computational complexity of the Gr-Gen-SM system can be expressed as [15]

$$
\begin{aligned}
\Gamma_{C_{G r-G e n-S M}=(}= & N_{G} N_{r}(2(N u-1)) \\
& \left.+4 N_{r}\right)(2)^{\eta_{G r-G e n-S M}}
\end{aligned}
$$

In the case of the proposed F-Gr-Gen-SM, each group is considered as F-Gen-SM system, which requires at most $\left(\left\lceil\frac{N_{G t}}{2}-1\right\rceil\right)$ complex summations for all $N_{t G}$ equalling at least 3 transmit antennas. Hence, each group is considered as an element in a MIMO system. Thereby, the number of required operations for the proposed scheme can be expressed as

$$
\begin{aligned}
\Gamma_{C_{F-G r-G e n-S M}=} & \left(4 N_{G} N_{r}\left(2\left\lceil\frac{N_{G t}}{2}-1\right\rceil\right)\right. \\
& \left.+4 N_{r}\right)(2)^{\eta_{F-G r-G e n-S M}}
\end{aligned}
$$

\section{SIMULATION RESULTS}

Using Monte Carlo simulations, the performance of the proposed scheme was evaluated. Three performance metrics; namely, achievable data rate, ABER, and computational complexity, are considered. First, for different $N_{t}$, the F-Gr-Gen-SM achievable data rates are compared with that of the classical SM in [1], Gen-SM in [7], F-Gen-SM in [13], and Gr-Gen-SM in [15]. Then, the ABER of the proposed F-Gr-Gen-SM is evaluated and compared with that of the conventional SMTs using various values of M-order QAM. Finally, the complexity of the F-Gr-Gen-SM scheme is evaluated and compared with the complexity of different SMTs.

\subsection{ACHIEVABLE DATA RATE}

Maximum achievable data rate of the proposed scheme (5) is evaluated and compared with that of SM (1), Gen-SM (2), F-Gen-SM (3), and Gr-Gen-SM (4) for various numbers of $N_{t}$. It is worthy of mentioning that the employed modulation techniques are 4-QAM, 8QAM, and 16-QAM. For both Gr-Gen-SM and proposed F-Gr-Gen-SM, the number of groups is assumed to equal 2. Also, for Gen-SM, the number of activated transmit antennas equals 2 , (i.e. $N_{u}=2$ ). If $N_{u}$ increases, the probability that the antenna index is repeated over different antenna subsets will increase, which in turn will degrade the error performance of the conventional GenSM. Therefore, $\mathrm{N}_{\mathrm{u}}=2$ is assumed [7].

Figure 2 plots the maximum achievable data rate versus $N_{t}$. Fig. 2 clearly shows that when $N_{t}$ increases, the achievable data rate increases. At a lower $N_{t}$ values, there are no significant differences in the achievable data rates among the different SMTs. Whereas at a large number of transmit antennas, our proposed scheme outperforms the other existing scheme. For example, when $N_{t}=22$, our proposed scheme superior to the existing scheme by at least $43 \%$.

Figure 3 plots the maximum achievable data rate versus $N_{t}$. In Fig. 3 , it is also clearly shown that increasing the order of QAM modulation means more achievable data rate for all schemes, but with different amounts. Especially for Gr-Gen-SM and the proposed scheme, the increase is greatly significant because each group operates as a separate unit with a separate digital modulation and thus the multiplexing gain contains the gain of M-QAM modulation.

Figure 4 confirms the above result where the data rates increase with the increase in the M-order of QAM. Also, our proposed scheme achieves the maximum data rates in comparison with the other different SMTs. In general, the proposed F-Gr-Gen-SM outperforms all other SMTs in all cases except one case, which is when $N_{G t}=2$. In this case, the achievable data rates of both the proposed and Gr-Gen-SM schemes are equals. On contrast, as $N_{G}$ increases to the maximum possible number, the advantages gained by the SMTs are reduced and these techniques become closer to the MIMO technique.

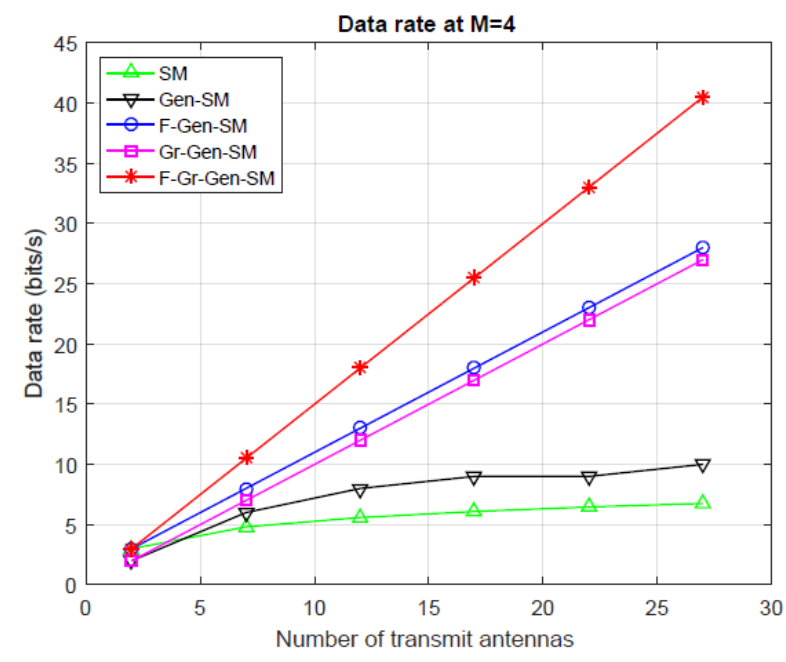

FIGURE 2. The maximum achievable data rates versus $N_{t}$, for $\mathrm{M}=4$. 


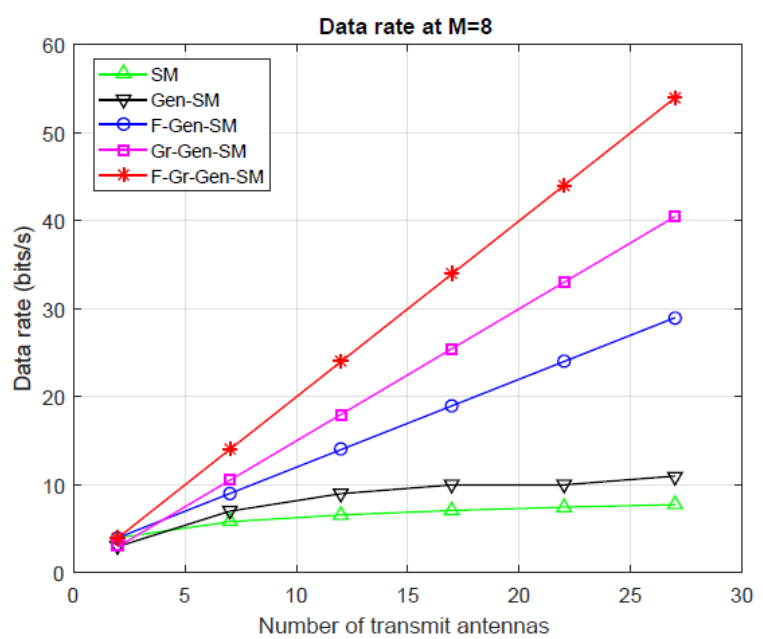

FIGURE 3. The maximum achievable data rates versus $N_{t}$, for $M=8$.

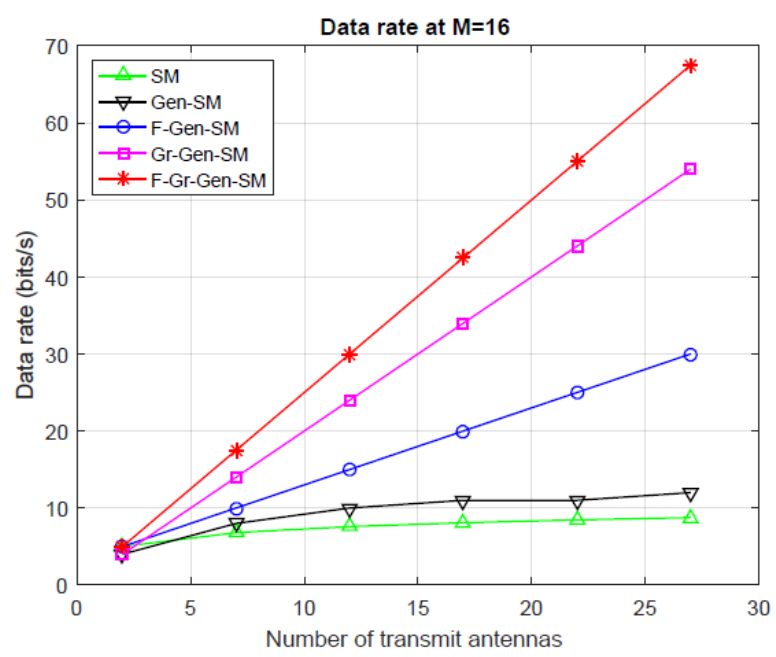

FIGURE 4. The maximum achievable data rates versus $N_{t}$, for $M=16$.

In Fig. 5, Fig. 6 and Fig. 7, the maximum achievable data rates are calculated using 4, 16, 32 and 64 transmit antennas under a different order of QAM. These charts clearly show that the spectral efficiency of the SMTs depends on the order of QAM modulation and $N_{t}$.

In Fig. 5, the spectral efficiency of the proposed scheme is compared with F-Gen-SM and Gr-Gen-SM schemes using $N_{t}=4,16,32$ and 64 antennas and 8QAM. Our proposed scheme always superior to other schemes and the difference widens with other schemes whenever the number of transmit antennas increased.

The Gr-Gen-SM scheme comes second especially with a high number of transmit antennas. For both Gr-GenSM and our proposed scheme, we choose a moderate number of groups to get high spectral efficiency with acceptable requirements.

The number of groups and antennas in each group are $2 \times 2,4 \times 4,8 \times 4$ and $8 \times 8$ for $4,16,32,64$ total transmit antennas, respectively.

In Fig. 6, the spectral efficiency of the proposed F-GrGen-SM is weighted against F-Gen-SM and Gr-Gen-SM schemes using $N_{t}=4,16,32$ and 64 antennas under 16-
QAM digital modulation. As shown, our proposed scheme continues to prevail. The Gr-Gen-SM is still ranking second. For both Gr-Gen-SM and our proposed scheme, the same consideration is taken as in Fig. 5.

In Fig. 7, our proposed scheme outperforms other schemes regardless of the value of $N_{t}$. Under 32-QAM digital modulation, the spectral efficiency of the proposed scheme is compared with F-Gen-SM and GrGen-SM. This figure explains that regardless the value of $N_{t}$ or the order of QAM, our proposed scheme achieves the best spectral efficiency weighted against other SMTs schemes.

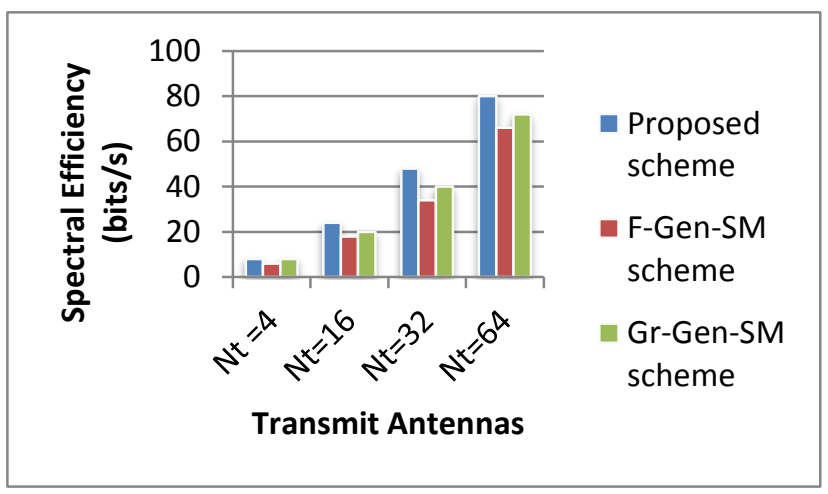

FIGURE 5. The spectral efficiency of the proposed F-GrGen-SM compared with the spectral efficiency of F-GenSM [13] and Gr-Gen-SM [15] at 8-QAM with different $N_{t}$.

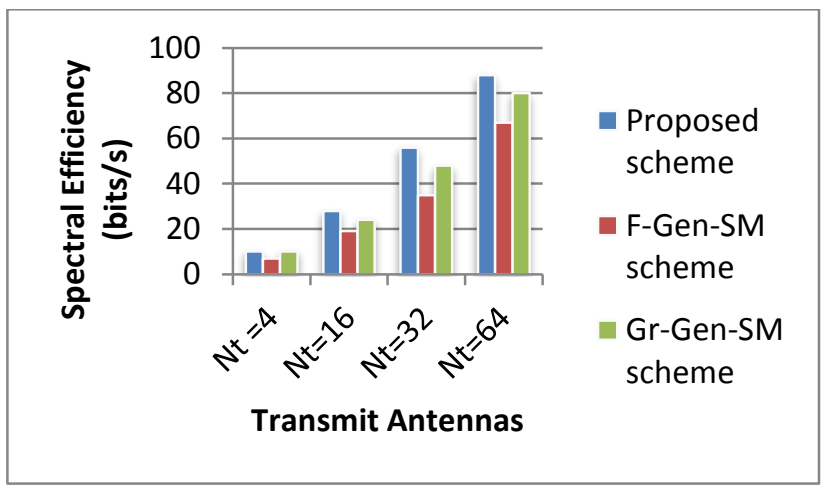

FIGURE 6. The spectral efficiency of the proposed F-GrGen-SM compared with the spectral efficiency of F-GenSM [13] and Gr-Gen-SM [15] at 16-QAM with different $N_{t}$.

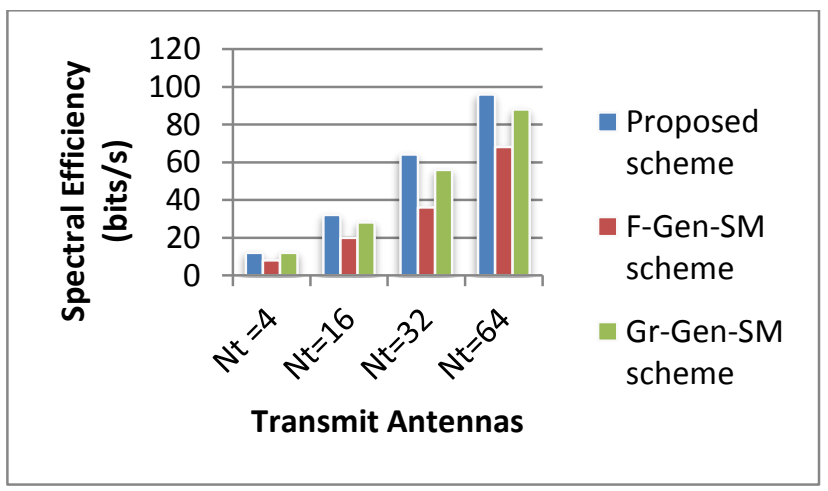

FIGURE 7. The spectral efficiency of the proposed F-GrGen-SM compared with the spectral efficiency of F-GenSM [13] and Gr-Gen-SM [15] at 32-QAM with different $\boldsymbol{N}_{t}$. 
TABLE 2. The number of required $N_{t}$ of the different SMTS using 4-QAM to achieve 10 bpcu.

\begin{tabular}{|c|c|c|c|c|c|}
\hline $\begin{array}{c}\text { Space } \\
\text { Modulation } \\
\text { technique }\end{array}$ & SM & $\begin{array}{c}\text { Gen- } \\
\text { SM }\end{array}$ & $\begin{array}{c}\text { F- } \\
\text { Gen- } \\
\text { SM }\end{array}$ & $\begin{array}{c}\text { Gr- } \\
\text { Gen- } \\
\text { SM }\end{array}$ & $\begin{array}{c}\text { F-Gr- } \\
\text { Gen- } \\
\text { SM }\end{array}$ \\
\hline$N_{t}$ & 256 & 11 & 9 & 10 & 8 \\
\hline$N_{G}$ & - & - & - & 2 & 2 \\
\hline$N_{u}$ & - & 5 & - & 2 & - \\
\hline
\end{tabular}

TABLE 3. The number of required $N_{t}$ of the different SMTS using 8-QAM to achieve $10 \mathrm{bpcu}$.

\begin{tabular}{|c|c|c|c|c|c|}
\hline $\begin{array}{c}\text { Space } \\
\text { Modulation } \\
\text { technique }\end{array}$ & SM & $\begin{array}{c}\text { Gen- } \\
\text { SM }\end{array}$ & $\begin{array}{c}\text { F- } \\
\text { Gen- } \\
\text { SM }\end{array}$ & $\begin{array}{c}\text { Gr- } \\
\text { Gen- } \\
\text { SM }\end{array}$ & $\begin{array}{c}\text { F-Gr- } \\
\text { Gen- } \\
\text { SM }\end{array}$ \\
\hline$N_{t}$ & 128 & 10 & 8 & 8 & 6 \\
\hline$N_{G}$ & - & - & - & 2 & 2 \\
\hline$N_{u}$ & - & 5 & - & 2 & - \\
\hline
\end{tabular}

TABLE 4. The number of required $N_{t}$ of the different SMTS using 16-QAM to achieve $10 \mathrm{bpcu}$.

\begin{tabular}{|c|c|c|c|c|c|}
\hline $\begin{array}{c}\text { Space } \\
\text { Modulation } \\
\text { technique }\end{array}$ & SM & $\begin{array}{c}\text { Gen- } \\
\text { SM }\end{array}$ & $\begin{array}{c}\text { F- } \\
\text { Gen- } \\
\text { SM }\end{array}$ & $\begin{array}{c}\text { Gr-Gen- } \\
\text { SM }\end{array}$ & $\begin{array}{c}\text { F-Gr- } \\
\text { Gen- } \\
\text { SM }\end{array}$ \\
\hline$N_{t}$ & 64 & 8 & 7 & 4 & 4 \\
\hline$N_{G}$ & - & - & - & 2 & 2 \\
\hline$N_{u}$ & - & 4 & - & 1 & - \\
\hline
\end{tabular}

On the other hand, with certain values of the achievable data rates and the order of QAM modulation, comparisons in terms of $N_{t}$ are illustrated between SM, Gen-SM, F-Gen-SM, Gr-Gen-SM and F-Gr-Gen-SM in Table 2, Table 3, and Table 4. As shown in Table 2, to achieve 10 bpcu using 4-QAM, our proposed scheme is the best one in reducing the required number of transmit antennas (8 transmit antennas). F-Gen-SM requires 9 transmit antennas. Gr-Gen-SM comes third as $N_{t}=10$. And the Gen-SM uses $N_{t}=11$ with 5 activated transmit antennas at a time. The conventional SM is the worst scheme in terms of saving the required transmit antennas as it needs 256 transmit antennas to achieve the same data rates. In another meaning, our proposed scheme saves more than $96 \%$ of $N_{t}$ of the conventional SM, $26 \%$ of $N_{t}$ of the Gen-SM, $11 \%$ of $N_{t}$ of F-Gen-SM and $20 \%$ of $N_{t}$ are saved as compared with Gr-Gen-SM.

Results in Table 3 show that to achieve $10 \mathrm{bpcu}$ using 8-QAM, our proposed scheme also is the optimum one in terms of saving the required transmit antennas (6 transmit antennas). Both F-Gen-SM and Gr-Gen-SM require 8 transmit antennas, and the Gen-SM requires 10 transmit antennas. The conventional SM comes last as it needs 128 transmit antennas to achieve similar data rates. In other words, the proposed scheme saves more than $95 \%$ of the transmit antennas of the conventional SM, $40 \%$ of the transmit antennas of the Gen-SM and $25 \%$ of the transmit antennas of both F-Gen-SM and GrGen-SM.

Finally, Table 4 again confirms the advantage of the proposed scheme. Also, the Gr-Gen-SM improved with increasing the order of the QAM. In other words, our proposed scheme economizes the required transmit antennas.

Our proposed scheme saves almost $93 \%$ of the required $N_{t}$ of SM. While, compared with Gen-SM, the proposed scheme provides $50 \%$ of the required transmit antennas to achieve the same data rates. Also, F-Gr-Gen-
SM provides almost $42 \%$ of the F-Gen-SM in terms of the required transmit antennas. Due to the equal number of $N_{G t}$ for both Gr-Gen-SM and F-Gr-Gen-SM, both schemes require the same $N_{t}$.

\subsection{ABER PERFORMANCE}

In this section, the ABER of the proposed scheme is evaluated using numerical simulations. Using the union bounding technique, the upper-bound for the ABER can be obtained [16]-[18]. This can be simulated using the Monte Carlo method, where every single value of BER is computed by averaging at least one million transmissions of symbol over different Rayleigh fading channel coefficients.

Fig. 8, Fig. 9, and Fig. 10 describe the ABER comparison considering the simulation parameter as in Table 2, Table 3, and Table 4, respectively. Note that, there are 4 antennas are employed at the receiving side.

As in Fig. 8, the excellence of the proposed scheme is clearly shown when is weighted against F-Gen-SM and Gr-Gen-SM. For a target ABER of $10^{-2}$, the F-Gr-GenSM scheme outperforms both the Gr-Gen-SM and FGen-SM systems by using about $5 \mathrm{~dB}$ and $6 \mathrm{~dB}$ lower SNR values, respectively. And at an ABER value of $10^{-3}$, the F-Gr-Gen-SM scheme also outperforms both the Gr-Gen-SM and F-Gen-SM systems by approximately $4.7 \mathrm{~dB}$ and $4.6 \mathrm{~dB}$, respectively.

Likewise, the ABER comparison using 8-QAM between F-Gen-SM, Gr-Gen-SM, and F-Gr-Gen-SM, is presented in Fig. 9. For an ABER of $10^{-2}$, the proposed scheme offers a performance improvement of around 2.4 $\mathrm{dB}$ and $7.5 \mathrm{~dB}$ over the Gr-Gen-SM and F-Gen-SM systems, respectively. Also, the proposed scheme is able of gaining a SNR of $4 \mathrm{~dB}$ and $5 \mathrm{~dB}$ over both Gr-GenSM and F-Gen-SM schemes, respectively at an ABER of $10^{-3}$.

Finally, the ABER comparison between F-Gen-SM, Gr-Gen-SM, and F-Gr-Gen-SM using 16-QAM is depicted in Fig. 10. For an ABER of $10^{-2}$, Gr-Gen-SM scheme outperforms both the F-Gr-Gen-SM and F-GenSM schemes by about $4.5 \mathrm{~dB}$ and $11 \mathrm{~dB}$, respectively.

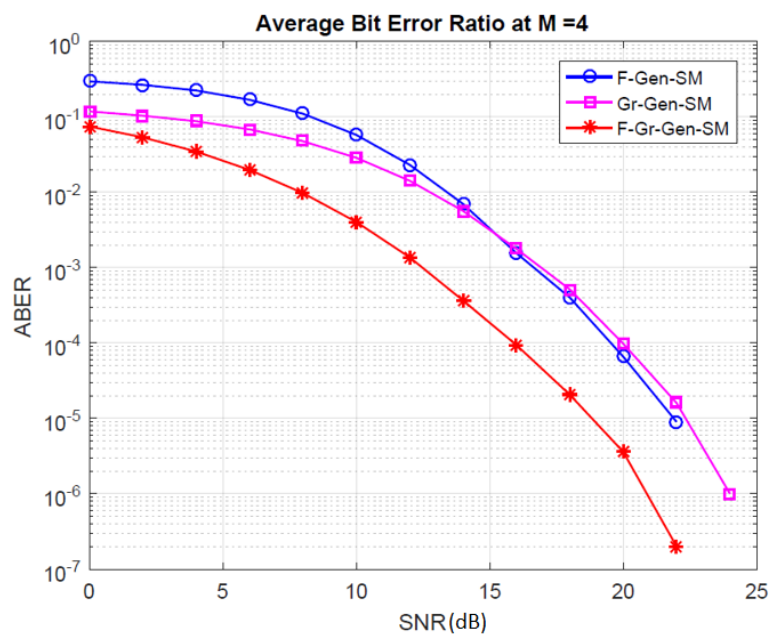

FIGURE 8. The ABER performance of the proposed FGr-Gen-SM compared with the ABER performance of $F$ Gen-SM [13] and Gr-Gen-SM [15] at 4-QAM with different $N_{t}$. 




FIGURE 9. The ABER performance of the proposed FGr-Gen-SM compared with the ABER performance of $F$ Gen-SM [13] and Gr-Gen-SM [15] at 8-QAM with different $N_{t}$.

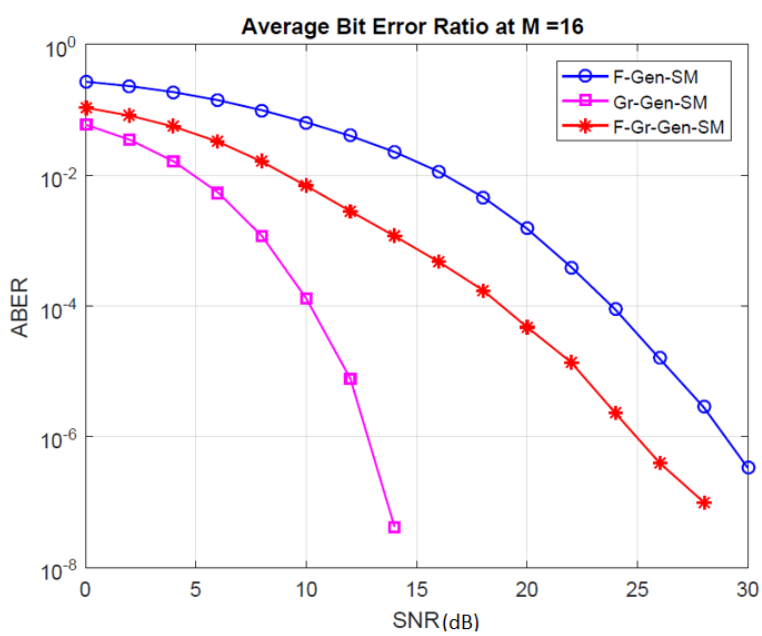

FIGURE 10. The ABER performance of the proposed FGr-Gen-SM compared with the ABER performance of $F$ Gen-SM [13] and Gr-Gen-SM [15] at 16-QAM with different $N_{t}$

And for an ABER value of $10^{-3}$, Gr-Gen-SM scheme outperforms both F-Gr-Gen-SM and F-Gen-SM by almost $6.5 \mathrm{~dB}$ and $11.6 \mathrm{~dB}$, respectively.

In general, with the same considerations, the F-GenSM scheme is negatively affected by high values of the SNR, while our proposed scheme suffers from ABER at high M-order of QAM modulation.

At a constant value of data rate, $N_{t}$ is inversely proportional to the order of QAM.

At higher order of M-QAM, the Gr-Gen-SM scheme activates the lowest number of $N_{G t}$, which in turn achieves the best ABER values compared with other SMTs.

\subsection{COMPUTATIONAL COMPLEXITY}

This subsection focuses on calculating the computational complexity at the receiving side of the F-Gr-Gen-SM using (14) and comparing it with its counterparts of the
Mux-MIMO, SM, Gen-SM, F-Gen-SM, and Gr-Gen-SM provided by (9), (10), (11), (12), and (13) respectively.

The computational complexities of these different SMTs are compared under two different scenarios.

\subsubsection{SCENARIO 1: DIFFERENT ORDERS OF MODULATION AND THE SAME $\boldsymbol{N}_{\boldsymbol{t}}$}

Using the same $N_{t}$ and different orders of QAM, the complexity comparisons of different SMTs are shown in Figs. 11, 12, 13, and 14.

As shown in Fig. 11, a comparison in complexity between the F-Gr-Gen-SM, Gr-Gen-SM, F-Gen-SM, and MIMO schemes using 4-QAM modulation. Although the proposed scheme suffers from additional computational complexity compared with Gr-Gen-SM and F-Gen-SM, it has a significant improvement in computational complexity as compared with the conventional MIMO scheme.

Due to the high computational complexity of MIMO than other SMTs, MIMO scheme will be out of comparison in Figs 12, 13 and 14.

Therefore, for a more accurate comparison, Fig. 12, Fig. 13, and Fig. 14 show complexity comparison between the Gr-Gen-SM, F-Gen-SM, and the proposed F-Gr-Gen-SM at $\mathrm{M}=4,8$, and 16 , respectively. For GrGen-SM and F-Gr-Gen-SM scheme considering each group contains 3 transmit antennas and $N_{u}=2$ for each group in the Gr-Gen-SM scheme.

In Fig. 12, complexity of F-Gr-Gen-SM is calculated and compared with that of both Gr-Gen-SM and F-GenSM schemes using 4-QAM.

As both the spectral efficiency and the number of active antennas of any scheme play an essential role in the calculation of the computational complexity while considering the same $N_{t}$ for all schemes, F-Gr-Gen-SM suffer from high values of $\Gamma_{C}$ when compared with other different SMTs. F-Gen-SM scheme comes second in terms of data rates; thus, it has a better computational complexity performance than the proposed F-Gr-GenSM.

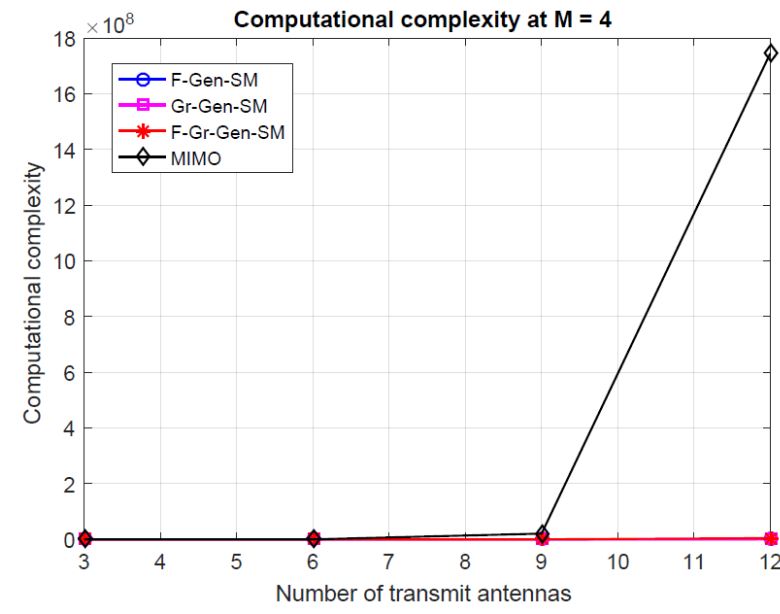

FIGURE 11. The computational complexity of the proposed F-Gr-Gen-SM compared with the computational complexity of F-Gen-SM [13], Gr-Gen-SM [15] and MIMO [19] at 4-QAM with different $N_{t}$ using 4 receive antennas. 
The best performance of the computational complexity using the ML detection method and 4-QAM is achieved by the Gr-Gen-SM scheme.

The same previous comparison using 8-QAM is illustrated in Fig. 13, which also confirms that the complexity of the proposed scheme is the main disadvantages of this scheme. Using 8-QAM, the spectral efficiency of the Gr-Gen-SM precedes that of the F-Gen-SM, so the computational complexity of the Gr-Gen-SM increased.

Fig. 14 confirms that the complexity of the proposed scheme needed to be improved using an advanced detection method.

In general, more increase in the order of QAM leads to higher spectral efficiency, which means more received bits that are causing more complexity.

This is clearly shown in Gr-Gen-SM scheme, which its complexity is getting worse by increasing the order of QAM modulation.

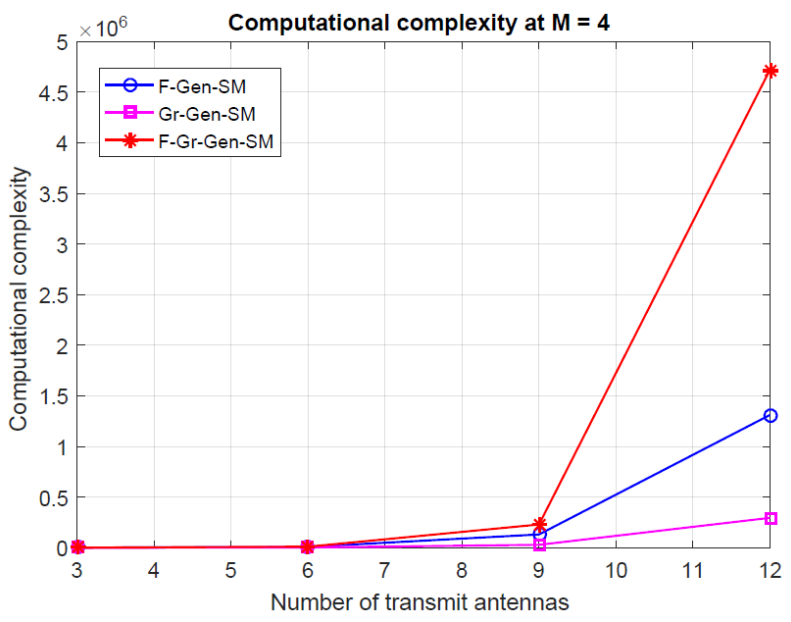

FIGURE 12. The computational complexity of the proposed F-Gr-Gen-SM compared with the computational complexity of F-Gen-SM [13] and Gr-Gen-SM [15] at 4-

QAM with different $N_{t}$ using 4 receive antennas.

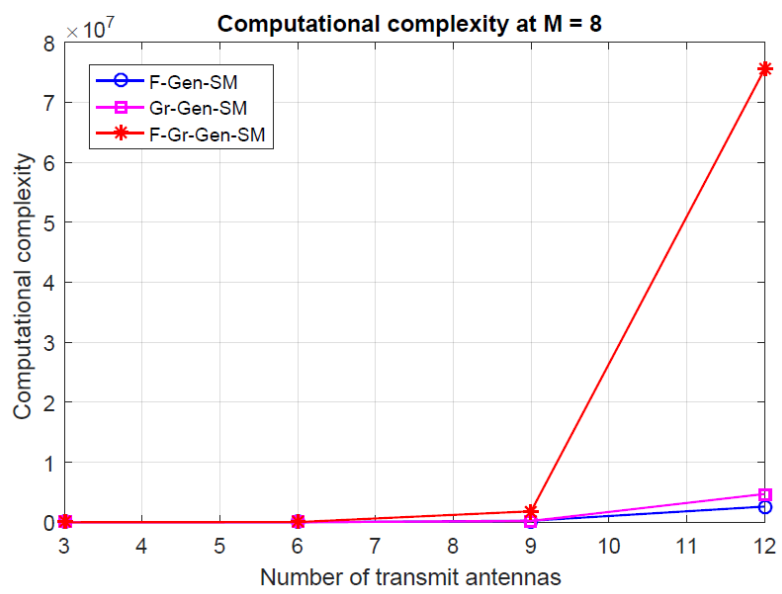

FIGURE 13. The computational complexity of the proposed F-Gr-Gen-SM compared with the computational complexity of F-Gen-SM [13] and Gr-Gen-SM [15] at 8QAM with different $N_{t}$ using 4 receive antennas.

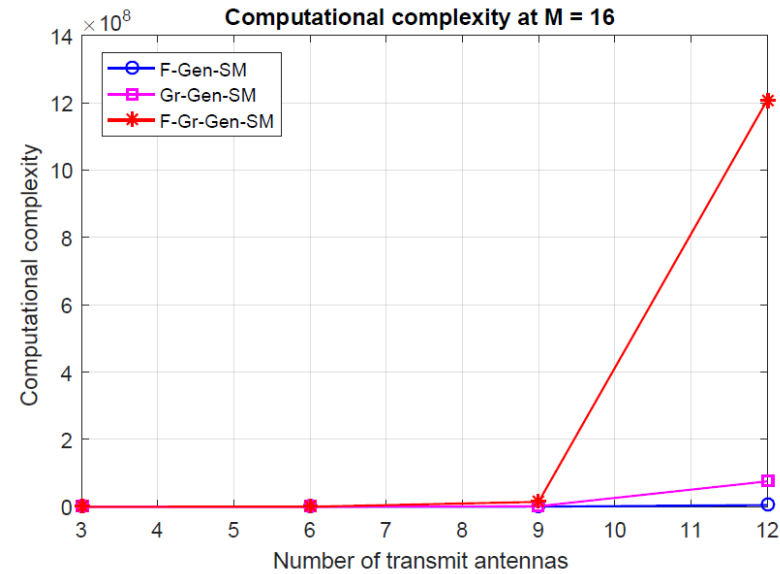

FIGURE 14. The computational complexity of the proposed F-Gr-Gen-SM compared with the computational complexity of F-Gen-SM [13] and Gr-Gen-SM [15] at 16-

QAM with different $N_{t}$ using 4 receive antennas.

Using 4-QAM, Gr-Gen-SM is the best scheme in terms of computational complexity, whereas it is no longer the best using a higher order of QAM. This is because of the spectral efficiency of Gr-Gen-SM that is described by (4), which is the most affected spectral efficiency by M-order of QAM.

\subsubsection{SCENARIO 2: THE SAME ORDER OF MODULATION AND DIFFERENT $\boldsymbol{N}_{t}$}

In Table 5, a numerical comparison in terms of computational complexity between our proposed scheme and SM, Gen-SM, F-Gen-SM, and MIMO schemes is evaluated using 4-QAM and different numbers of transmit antennas to achieve ( $8 \mathrm{bpcu}$ ) data rate.

The computational complexity of our proposed scheme comes second, while SM is the best. This is because only one transmit antenna is activated for the SM scheme.

Hence, $N_{t}$ is not taken into account for the SM scheme in contrast to other SMTs schemes.

TABLE 5. $\Gamma_{C}$ of different SMTS at 8 bpcu.

\begin{tabular}{|c|c|c|c|c|c|}
\hline $\begin{array}{c}\text { Space } \\
\text { Modulation } \\
\text { technique }\end{array}$ & SM & $\begin{array}{c}\text { Gen- } \\
\text { SM }\end{array}$ & $\begin{array}{c}\text { F-Gen- } \\
\text { SM }\end{array}$ & $\begin{array}{c}\text { F-Gr- } \\
\text { Gen-SM }\end{array}$ & MIMO \\
\hline$N_{t} \times N_{r}$ & $64 \times 4$ & $8 \times 4$ & $7 \times 4$ & $6 \times 4$ & $4 \times 4$ \\
\hline $\begin{array}{c}\text { Computational } \\
\text { complexity }\end{array}$ & 8192 & 49152 & 49152 & 20480 & 20480 \\
\hline
\end{tabular}

\section{CONCLUSION}

In this paper, F-Gr-Gen-SM scheme was presented to achieve a high spectral efficiency compared with different SMTs, while maintaining the advantages of SMT as much as possible. Based on the idea of antennas grouping, we propose a F-Gr-Gen-SM system. According to the proposed system, the antenna grouping increases the spectral efficiency by multiplexing the data from all groups. In detail, unlike Gr-Gen-SM, which aiming for increasing the spectral efficiency by maximizing the number of groups, our proposed scheme uses a moderate number of groups and compensates for this decrease in spectral efficiency by implementing the F-Gen-SM scheme in each group. As each group 
transmits different data and by decreasing $N_{G}$ for F-GrGen-SM scheme, the ICI and the number of required RF chains will be decreased compared with the MuxMIMO. But the SM scheme remains the best in terms of ICI elimination and it needs fewer RF chains. Thus, the cost and complexity of F-Gr-Gen-SM are decreased as compared with the Gr-Gen-SM. So, the proposed scheme is considered as a trade-off between SM and Mux-MIMO scheme.

To achieve spectral efficiency of $10 \mathrm{bpcu}$, our proposed scheme saves at least $11 \%$ of the required transmit antennas compared with the different SMTs, using 4-QAM. While using 8-QAM, the proposed scheme saves $25 \%$ of the required transmit antennas at least compared with the different SMTs. Note that, setting two transmit antennas for each group, is the only case in which the proposed and Gr-Gen-SM schemes achieve the same performance as F-Gen-SM is considered as Gen-SM when the transmit antennas are 2 antennas. The ABER performance and computational complexity have been analyzed and compared with different SMTs in this paper.

Simulation results showed that F-Gr-Gen-SM scheme has a good impression through the spectral efficiency and the ABER for different values of SNR, while a detection method with low complexity is required for this scheme.

\section{Credit Authorship Contribution Statement:}

Islam E. Shaalan: Conceptualization, Formal analysis, Investigation, Methodology, Supervision;

Saied Dawod: Conceptualization, Data curation, Formal analysis, Software, Writing - original draft;

Sherif M. Abuelenin: Writing - review \& editing Supervision.

\section{Declaration of competing Interest}

The authors declare that they have no known competing financial interests or personal relationships that could have appeared to influence the work reported in this paper.

\section{References}

[1] R. Mesleh, H. Haas, S. Sinanović, C. W. Ahn, and S. Yun, "Spatial modulation," IEEE Transactions on Vehicular Technology, vol. 57, no. 4, pp. 22282241, July 2008.

[2] E. S. Telatar, "Capacity of multi-antenna gaussian channels," European Transaction on Telecommunication, vol. 10, No. 6, pp. 585-595, November-December 1999.

[3] J. Jeganathan, A. Ghrayeb, L. Szczecinski, and A. Ceron, "Space shift keying modulation for MIMO channels," IEEE Transactions Wireless Communications, vol. 8, no. 7, pp. 3692-3703, July 2009.

[4] M. Di Renzo and H. Haas, "Space shift keying (SSK) modulation with partial channel state information: Optimal detector and performance analysis over fading channels," IEEE Transactions Communications, vol. 58, no. 11, pp. 3196-3210,
November 2010.

[5] J. Jeganathan, A. Ghrayeb, and L. Szczecinski, "Generalized space shift keying modulation for MIMO channels," in Proc. IEEE 19th International Symposium on Personal, Indoor and Mobile Radio Communications, Cannes, France 15-18 September 2008, pp. 1-5.

[6] K. Ntontin, M. Di Renzo, A. I. Perez-Neira, and C. Verikoukis, "Adaptive generalized space shift keying," EURASIP Journal on Wireless Communications and Networking, vol. 2013, no. 43, pp. 1-15, Febreuary 2013.

[7] A. Younis, N. Serafimovski, R. Mesleh, and H. Haas, "Generalised spatial modulation," in Proc. the 44th Annual Asilomar Conference on Signals, Systems, and Computers (ASILOMAR), Pacific Grove, California, USA, 7-10 November 2010, pp. 1498-1502.

[8] A. Younis, D. A. Basnayaka, and H. Haas, "Performance analysis for generalised spatial modulation," in Proc. 20th European Wireless Conference, Barcelona, Spain, May 14-16 2014, pp. $1-6$.

[9] T. Datta and A. Chockalingam, "On generalized spatial modulation," in Proc. IEEE Wireless Communications and Networking Conference (WCNC), Shanghai, China, 7-10 April 2013, pp. 2716-2721.

[10] A. Zaki, M. Nassar, M. Aly, W. Badawi, "Generalized Spatial Modulation System Using Massive MIMO Space Time Coding Antenna Grouping. "Entropy journal, vol. 22 no. 12, pp. 1350, 2020.

[11] H. Bitra, and P. Ponnusamy, "Performance Analysis of Adaptive Generalized Spatial Modulation." 2020 International Conference on Artificial Intelligence and Signal Processing (AISP), Amaravati, India, IEEE, 10-12 Jan. 2020.

[12] H. S. Hussein and M. Elsayed, "Fully-quadrature spatial modulation," in Proc. IEEE International Black Sea Conference on Communications and Networking (BlackSeaCom), Batumi, Georgia, 4-7 June 2018, pp. 1-5.

[13] M. Elsayed, H. S. Hussein, and U. S. Mohamed, "Fully generalised spatial modulation," in Proc. 35th National Radio Science Conference (NRSC), Cairo, Egypt, 20-22 March 2018, pp. 274-282.

[14] H. S. Hussein, M. Elsayed, U. S. Mohamed, H. Esmaiel, E. M. Mohamed, "Spectral Efficient Spatial Modulation Techniques", Access IEEE, vol. 7, pp. 1454-1469, 2019.

[15] F. R. Castillo- Soria, J. Cortez- González, R. Ramirez- Gutierrez, F. M. Maciel- Barboza, and L. Soriano- Equigua, "Generalized quadrature spatial modulation scheme using antenna grouping," ETRI Journal, vol. 39, no. 5, pp. 707-717, October 2017.

[16] M. K. Simon and M. S. Alouini, Digital Communication Over Fading Channels, 2nd ed., vol. 95, Hoboken, NJ, USA: John Wiley \& Sons, 
2005.

[17] J. G. Proakis, Digital Communications, 4th ed., New York, NY, USA: McGraw-Hill, 1995.

[18] M. S. Alouini, A. J. Goldsmith "A unified approach for calculating error rates of linearly modulated signals over generalized fading channels," IEEE Transactions on Communications, vol. 47, no. 9, pp. 1324-1334, September 1999.

[19] R. Mesleh, and A. Alhassi, Space Modulation Techniques, John Wiley \& Sons, 2018. 